\title{
Les nouvelles aventures d'Alice : reprises et transfictions
}

\author{
Maryse Sullivan \\ Université d'Ottawa
}

Connue pour ses aventures au pays des merveilles, Alice est un personnage né dans la deuxième moitié du XIXe siècle qui, encore aujourd'hui, continue de jouir d'un succès littéraire et culturel. Il suffit de penser aux films (Alice in Wonderland [1951], Alice in Wonderland [2010]) et séries télévisées américains (Once Upon a Time [2011 - ], Once Upon a Time in Wonderland [2013-2014]) ou aux nombreuses suites, adaptations et réécritures de ses aventures (notamment les livres Le Pays des 36000 volontés, Le Sourire du chat, Alice in Wonderland High, Alice au pays des embrouilles! et les cycles Tales from Wonderland et The White Rabbit Chronicles) pour 
saisir l'influence et l'omniprésence de cette fillette britannique. Alice et les farfelus personnages des romans de Carroll ont été récupérés et réappropriés maintes fois pour leur faire vivre de nouvelles aventures dans d'autres univers. Grâce à la transfictionnalité, une notion développée par Richard SaintGelais pour décrire le phénomène qui se produit lorsque des éléments de différentes fictions se trouvent transplantés et combinés (2011, p. 314-315), de nouveaux lecteurs peuvent (re)découvrir Alice, le Chat du comté de Chester, le Chapelier fou ou la Reine de cœur. En d'autres termes, ce phénomène survient chaque fois qu'un personnage d'une fiction précise s'infiltre dans un autre univers diégétique. Une transfiction est le produit d'un croisement entre deux ou plusieurs mondes fictionnels (Saint-Gelais, 2011, p. 314-315). Par exemple, dans son roman Time for Sherlock Holmes, David Dvorkin met en scène le célèbre Sherlock Holmes de Conan Doyle, mais le transpose dans l'univers de La Machine à explorer le temps de H.G Wells. Le roman place une intrigue novatrice dans un monde qui se veut le mélange de deux diégèses provenant d'œuvres et d'auteurs distincts. De la même manière, de nombreuses transfictions reprennent Alice, ce qui nous pousse à nous interroger sur le personnage, afin de mieux comprendre comment il est récupéré et présenté dans de nouvelles fictions et pourquoi il se prête bien à la réécriture et la transfictionnalité. Dans cette étude, nous analyserons l'histoire et le personnage d'Alice afin d'extraire la quintessence du personnage transfictionnel. Pour ce faire, nous dégagerons d'abord les conditions du personnage transfictionnel, c'est-àdire les caractéristiques de l'héroïne et de ses livres, qui facilitent les reprises et, parfois, les encouragent. Puis, dans la deuxième partie de notre étude, nous regarderons diverses 
transfictions contemporaines, provenant de genres et de médias distincts, qui s'intéressent à la fillette - La Bibliothécaire (roman jeunesse), Tales of Wonderland (roman graphique) et Once Upon a Time (télévision) - afin de comprendre comment l'histoire a été reprise par différents auteurs et transformée pour des publics variés.

\section{Les romans de Carroll : un carrefour de la tradition anglaise et un réservoir de héros}

Une des caractéristiques des livres d'Alice, soit Alice au pays des merveilles et De l'autre côté du miroir qui favorise les reprises est que ces romans sont eux-mêmes déjà des transfictions. Le pays des merveilles et le pays du miroir mêlent des personnages de la création de Carroll, telles les cartes de jeu ou les pièces d'échecs, avec des personnages qui existent déjà dans la tradition anglaise et l'imaginaire collectif des lecteurs grâce à des expressions populaires ou des nursery rhymes anglaises. En effet, au pays des merveilles, Alice fait la connaissance du personnage du Chat du comté de Chester, Cheshire cat, qui dérive de l'expression «to grin like a Cheshire cat ${ }^{1}$ » (Carroll, 1998, p. 309); elle rencontre aussi le Chapelier fou et le Lièvre de mars qui, eux, proviennent des expressions «mad as a hatter » et «mad as a March hare » (Carroll, 1990, p. 105 et $360)^{2}$. La Simili-Tortue, quant à elle, tient son nom d'une soupe

\footnotetext{
${ }^{1}$ "Sourire comme un chat du Cheshire », traduction de Jacques Papy (Carroll, 1990, p. 360).

2 « Fou comme un chapelier » et « fou comme un lièvre en mars », traductions de Jacques Papy (Carroll, 1990, p. 360).
} 
populaire «mock turtle soup» (Carroll, 1990, p. 134 et 362) 3 . Puis, au pays du miroir, Alice croise Tweedledee et Tweedledum (Carroll, 1990, chapitre 4), Humpty Dumpty (chapitre 6) et les personnages du Lion et de la Licorne (chapitre 7), qui émanent tous de comptines anglaises, un genre littéraire enfantin qui était demeuré populaire tout au long des XVIII" et XIXe siècles en Angleterre (Bouslough, 2007, p. 44) et que se sont approprié les enfants des classes privilégiées lors de l'essor de l'industrialisation au XIXe siècle (Escarpit, 2008, p. 47). À vrai dire, comme le conte travaillé et perfectionné de génération en génération par la transmission de bouche à oreille (Belmont, 1999, p. 232), les nursery rhymes et les chansons anglaises sont un héritage de la tradition orale, bien ancrée dans la culture du peuple (Opie, 1984, p. v-vi).

En intégrant des personnages que nous pourrions considérer déjà "imaginés", les mondes créés par Carroll deviennent ce que Richard Saint-Gelais appelle des forums transfictionnels qui sont " [une] forme extrême de croisement qui rassemble en un même récit, non pas deux (ou quelques) fictions préexistantes, mais la totalité du champ fictionnel, ou à tout le moins un sous-ensemble significatif de celui-ci » (2011, p. 222). Pour illustrer ce phénomène, Saint-Gelais donne en exemple l'œuvre L'Affaire D (1989) de Carlo Fruttero et Franco Lucentini, qui regroupe divers détectives fameux, comme Poirot, Maigret, Wolfe et Holmes, provenant de romans variés, dans l'espoir de résoudre une grande enquête. De même, la série de bandes dessinées The League of Extraordinary Gentlemen (1999 - ) réunit divers héros d'œuvres romanesques,

\footnotetext{
${ }^{3}$ La traduction littérale signifie «Ersatz de soupe à la tortue », traduction de Jacques Papy (Carroll, 1990, p. 362).
} 
dont le capitaine Nemo, l'homme invisible de Wells (Hawley Griffin), Mina Harker de Dracula et d'autres protagonistes littéraires. Allant au-delà de l'intertextualité, les forums transfictionnels sont des lieux dans lesquels diverses fictions coexistent sur la base d'une communauté et non un collage ou une simple alliance de récits (Saint-Gelais, 2011, p. 224).

Carroll est plus proche de certains auteurs qui rassemblent des personnages provenant davantage de la tradition populaire que de la culture lettrée pour composer leur forum transfictionnel, comme Michel Tremblay, qui amalgame une dizaine de personnages de contes dans sa pièce Les Héros de mon enfance (1976). À ce sujet, il faut noter que les auteurs qui créent des forums transfictionnels avec des héros émanant de contes, de nursery rhymes, de chansons populaires ou du folklore ont souvent plus de liberté quant à la représentation des personnages, car ces derniers sont moins liés à un créateur précis. Moins fixés par un texte, les personnages provenant de la tradition sont davantage l'héritage d'une culture et d'un imaginaire collectif, c'est-à-dire d'un imaginaire qui, semblable à la mémoire collective, se définit comme un horizon de références marqué par l'histoire, la littérature, l'image et l'influence de la société, que partagent les membres d'un groupe précis et qui est parsemé de figures, de traditions et d'événements dont ils ont gardé le souvenir (Halbwachs, 1950, p. 103, 113 et 118; Popovic, 2008, p. 20). Les romans de Carroll s'inscrivent dans ce courant parce que les nursery rhymes tiennent leur succès de la transmission orale et parce que les auteurs de ces comptines, lorsqu'il y en avait, s'effaçaient devant les conteurs, qui étaient plus importants (Escarpit, 2008, p. 53). Alice au pays des merveilles et De l'autre côté du miroir sont alors des forums transfictionnels d'un sous-champ 
de la littérature anglaise bien ancré dans la tradition chantée du pays, qui inclut des expressions communes, des chansons populaires et des comptines ou nursery rhymes jouant sur les rimes et les répétitions (Prince, 2010, p. 155).

À vrai dire, plusieurs personnages mis en scène dans les livres de Carroll ne nécessitent aucune introduction, puisque l'expression ou la nursery rhyme de laquelle ceux-ci dérivent est connue par une majorité de lecteurs. Pour cette raison, afin que le lecteur puisse bien reconnaître le dicton du Cheshire cat par exemple, Carroll met beaucoup l'accent sur le sourire du chat dans le texte. Lorsqu'Alice demande à la duchesse pourquoi le chat sourit, elle répond : "C'est un chat du comté de Chester [...] voilà pourquoi. » (Carroll, 1990, p. 99) De cette façon, Carroll s'assure de mettre en scène non seulement le personnage, mais aussi son origine. Cette intégration facilite l'identification des personnages et permet aux lecteurs de se rappeler rapidement leur histoire. Alice croise aussi Humpty Dumpty lorsqu'il est " assis, les jambes croisées, à la turque, sur le faîte d'un mur très haut» (Carroll, 1990, p. 267), une action dépeinte dans les deux premiers vers de sa comptine célèbre ${ }^{4}$, ce qui accélère son identification.

La construction d'un forum transfictionnel, l'intégration de personnages déjà familiers ou déjà « imaginés » et, bien sûr, le succès dont ont profité les œuvres de Carroll semblent ainsi encourager les reprises puisqu'ils font en sorte que l'histoire et les personnages demeurent présents dans la mémoire des lecteurs. Depuis Carroll, le recyclage de personnages considérés « déjà épaissis, en quelque sorte, déjà constitués, déjà

\footnotetext{
${ }^{4}$ Humpty Dumpty sat on a wall, Humpty had a great fall (Carroll, 1998, p. 182).
} 
textualisés, participant à un imaginaire commun et traditionnel » (Prince, 2010, p. 92) est devenu un élément de plus en plus récurrent en littérature pour la jeunesse, selon Nathalie Prince, puisqu'il facilite et encourage la lecture du jeune lecteur.

L'intérêt que suscitent certains des personnages d'Alice au pays des merveilles ou De l'autre côté du miroir dépasse même le champ de la littérature enfantine et s'étend à plusieurs genres. Le personnage d'Alice a d'ailleurs été repris par plusieurs transfictions contemporaines dans divers genres, du roman érotique Alison's Wonderland (2010) au cycle de romans graphiques Tales from Wonderland (2007-2012) en passant par le cycle adolescent The White Rabbit Chronicles (2012-2014).

Une première caractéristique qui fait en sorte que cette fillette soit aussi récupérable est que le voyage lui est associé dans son texte d'origine, facilitant ses autres déplacements. Selon Isabelle Daunais, les personnages qui apparaissent déjà dans leur propre fiction comme "des êtres venus d'ailleurs" (2007, p. 355) se déplacent mieux et plus fréquemment que d'autres puisque cette caractéristique sert à les définir. Notamment, nous pouvons penser à Don Quichotte, au Petit Prince et, bien sûr, à Alice. Le voyage s'assimile à leur identité et contribue aux « retours » et à la gloire de ces personnages. Une deuxième caractéristique qui facilite l'infiltration de personnages dans de nouvelles fictions est leur malléabilité. Les héros doivent se renouveler tout en restant fidèles au monde qui les a immortalisés comme personnage (Daunais, 2007, p. 354-355 et 360). Il va sans dire que des héros célèbres, comme James Bond, Dracula ou Sherlock Holmes, qui demeurent présents dans nos esprits et dans la culture, sont 
populaires parce qu'ils sont malléables et parce qu'ils continuent de s'adapter aux publics, aux modes, à la technologie et aux époques (Bennett et Woollacott, 1987, p. 19-20). Quant à Alice, son imprévisibilité et le caractère onirique de ses aventures et des univers qui l'entourent encouragent ses renouvellements puisque plusieurs parties de son histoire demeurent encore voilées. De nouveaux auteurs peuvent alors travailler à recréer, à réinterpréter ou à élargir le pays des merveilles, le pays du miroir ou d'autres mondes modernes, en s'appuyant sur de nouvelles modes et de nouveaux publics. C'est ainsi que les auteurs réussissent à réanimer des personnages (illustres ou obscurs) en cherchant à les adapter et à les réinventer afin qu'ils puissent vivre une autre aventure (plus moderne), qui apporte quelque chose de nouveau à leur personnage et afin qu'ils puissent profiter d'une nouvelle ou d'une plus longue popularité auprès d'une autre génération et d'un autre public.

Alice et les personnages des romans de Carroll remplissent les conditions des personnages que nous pourrions qualifier de "transfictionnels» puisqu'ils ne cessent d'être réutilisés dans d'autres aventures et d'être accueillis par de nouveaux genres et de nouveaux publics. Dans la deuxième partie de cette étude, nous allons analyser trois transfictions modernes de genres et de médiums différents, soit $\mathrm{La}$ Bibliothécaire (1995) de Gudule, Tales from Wonderland (2010) de Raven Gregory, Joe Brusha et Ralph Tedesco, et l'épisode intitulé «Hat Trick» (2012) de la série télévisée Once Upon a Time des créateurs Adam Harowitz et Edward Kitsis, qui reprennent le personnage d'Alice afin de présenter son histoire à une autre génération, de la réécrire ou afin d'ajouter à celle-ci. 


\section{La Bibliothécaire}

Dans La Bibliothécaire, quatre adolescents décident d'aller à la bibliothèque en soirée pour repérer un livre magique, le grimoire, qu'ils croient pouvoir trouver dans les rayons pour la jeunesse. Lors de la soirée, les adolescents pénètrent, ou plutôt sautent, dans divers livres dans le dessein de localiser ce grimoire qui est lui-même peut-être logé dans un livre. Le premier des ouvrages dans lesquels ils plongent est Alice au pays des merveilles. Au moment où les héros guduliens atterrissent dans le roman de Carroll, il y a ce que Saint-Gelais appelle une "annexion » parce qu'un univers diégétique d'un autre auteur converge vers celui qu'a créé Gudule. L'annexion est le phénomène transfictionnel qui décrit ce qui se passe lorsqu'un auteur met en relation son univers diégétique avec autre univers établi, dans ce cas-ci celui de Carroll, et que cette fiction cohabite avec celle du nouvel auteur (2011, p. 200-201) ${ }^{5}$.

L'intrusion des héros guduliens, à savoir l'annexion, modifie l'œuvre originale de Carroll puisque l'ajout des protagonistes force les personnages d'Alice au pays des merveilles à réagir, ce qui entraîne certaines modifications narratives par rapport au récit initial et donne lieu à une nouvelle version. À ces moments, Gudule joue avec ce que SaintGelais appelle le «suspense transfictionnel», une préoccupation qui survient dans

\footnotetext{
5 Saint-Gelais donne en exemple l'opération qu'effectue Maurice Leblanc lorsqu'il mêle le monde qu'il a lui-même créé à celui de Conan Doyle dans Arsène Lupin contre Herlock Sholmès pour décrire l'annexion. (2011, p. 200 201). L'univers diégétique d'une œuvre connue se fusionne au nouvel univers.
} 
un récit où c'est la relation transfictionnelle elle-même, et plus précisément l'altération contrefictionnelle (possible) d'une intrigue préalable, qui deviennent matière à suspense, pour peu que le lecteur, alerté par quelque détail ou simplement soupçonneux, en vienne à se demander si c'est la même histoire qu'on est en train de lui raconter, ou bien quelque autre qui finira par en différer de façon plus ou moins importante (2011, p. 182-183).

Un suspense transfictionnel s'interpose dans le neuvième chapitre lorsque les héros atterrissent dans l'univers d'Alice au pays des merveilles parce que le récit de Gudule, c'est-à-dire la transfiction, suspend provisoirement l'issue déjà connue de l'histoire de Carroll et appuie l'hypothèse d'une possible bifurcation de l'intrigue pour ensuite laisser l'histoire reprendre son cours naturel (Saint-Gelais, 2011, p. 183). En effet, au moment où les cartes de jeu peignent les fleurs rouges et le cortège de la Reine de cœur s'avance (une action inscrite dans le roman original), les cartes remarquent les nouveaux personnages. La Reine de cœur se fâche d'ailleurs puisqu'Idda produit des «bruits incongrus» (Gudule, 1995, p. 89). Elle ordonne de couper la tête aux quatre personnages et devient furieuse lorsqu'ils se sauvent. Le lecteur vit un moment de suspense transfictionnel en observant la bifurcation possible de l'intrigue originale. S'il connaît Alice au pays des merveilles, le lecteur voit toutefois qu'après le départ du héros Guillaume et ses amis, l'histoire reprend rapidement son cours naturel puisque le cortège de la Reine ne part pas à leur poursuite, mais continue de s'intéresser aux jardiniers qui peignent les fleurs rouges comme dans le roman original (Gudule, 1995, p. 90).

Gudule présente alors une version différente de l'histoire connue, ce que Saint-Gelais appelle une «version transfictionnelle» (2011, p. 93). Une version transfictionnelle est une 
autre histoire avec des variantes, qui s'insère à l'intérieur de la zone temporelle déjà couverte par un récit initial. En autres mots, une version transfictionnelle est un nouveau récit qui reprend une "même» histoire (Saint-Gelais, 2011, p. 93-94). Même si le roman de Carroll suit son cours naturel et qu'il n'est pas altéré de façon majeure, la présence des nouveaux héros crée une interférence qui fait en sorte que «le nouveau récit rétroagi $[t]$ d'une manière perceptible sur le précédent » (SaintGelais, 2011, p. 140-141). L'influence des personnages, leurs actions et leurs commentaires donnent alors l'impression au lecteur que l'histoire est contée à nouveau, voire « retraversée », sous un nouvel angle, donnant naissance à une autre version. Cependant, même s'il s'agit d'une version transfictionnelle, Gudule reste fidèle au récit original et respecte l'ordre narratif (elle ne fait que des petites modifications), pour bien mettre en scène Alice au pays de merveilles et montrer à quoi ressemblerait une plongée dans le livre et dans l'univers romanesque de Carroll.

\section{Tales from Wonderland}

Dans les autres transfictions qui nous intéressent, les reprises se passent de façon différente. En effet, dans le roman graphique Tales from Wonderland, les auteurs nous présentent une version transformée des aventures d'Alice. Cette fois-ci, il s'agit d'une réécriture du deuxième roman de Carroll, De l'autre côté du miroir, et non d'une fusion avec Alice au pays des merveilles. Tales from Wonderland nous présente une version différente de l'histoire, plus noire et violente, davantage 
adaptée pour un public adulte, le public cible du roman graphique. Les auteurs Raven Gregory, Joe Brusha et Ralph Tedesco opèrent une translation temporelle en transposant l'action du roman anglais de l'époque victorienne dans un contexte moderne. Il s'agit d'une version transfictionnelle ou d'un hypertexte du roman de Carroll, qui cherche non seulement à créer des liens avec la fiction initiale, mais aussi à la modifier, en présentant au lecteur une autre version des faits. À ce sujet, Saint-Gelais indique notamment qu'« [u]ne transfiction peut coexister avec la fiction initiale en lui apportant diverses adjonctions, mais aussi, dans d'autres cas, se substituer à elle, ou du moins prétendre le faire, se donnant dès lors comme une nouvelle version» (2011, p. 139). Ici, les auteurs tentent de faire les deux, en essayant à la fois de forger des liens avec l'original et de créer un système de renvois entre autres en intégrant un jeu d'échecs, un miroir et les personnages du Roi et de la Reine rouge-, mais aussi de remplacer le texte initial en présentant une autre version. Contrairement au roman de Gudule, cette réécriture développe plus l'hypertextualité, à savoir une relation unissant les textes par imitation ou transformation, que la transfictionnalité, qui est une relation arrimant davantage les fictions grâce à une migration ou une modification de données diégétiques (SaintGelais, 2011, p.10-11). Il faut toutefois noter que, même s'ils modifient l'histoire et cherchent à la renouveler, Gregory, Busha et Tedesco essaient de respecter la construction du récit de Carroll et de reprendre généralement la même structure.

Carroll amorce le récit De l'autre du miroir avec Alice qui, enfermée lors d'une après-midi glaciale, décide de jouer avec ses chattes et d'imaginer une aventure. Elle dit "Faisons semblant », ce qui agit comme le catalyseur de l'aventure et qui 
la mène de l'autre côté du miroir du salon. Similairement, dans le premier épisode de Tales from Wonderland, la protagoniste cajole également un chat, qui serait réellement le Cheshire cat, et c'est lui qui l'encourage à traverser le miroir (Gregory, Busha et Tedesco, 2010, p. 24-28). Ensuite, après l'épisode avec la Reine rouge (Gregory, Busha et Tedesco, 2010, p. 30-49), le lecteur rencontre Tweedledee et Tweedledum (deux personnages de nursery rhymes), tout comme dans le récit original. Cependant, au lieu de raconter à Alice l'histoire du Morse et du Charpentier comme dans l'hypotexte; dans le roman graphique, Tweedledee et Tweedledum croisent plutôt ces deux personnages dans un restaurant où ils découvrent que le Charpentier ne trompe plus seulement des huîtres pour les manger - comme c'est le cas dans le roman de Carroll et aussi dans le film de Disney d'Alice au pays des merveilles (1951) - , mais qu'il est maintenant devenu un meurtrier. Les dernières planches de l'épisode nous montrent d'ailleurs le Charpentier en train d'assassiner le Morse et une image des corps inanimés de Tweedledee et Tweedledum, qui ont aussi été pris au piège (Gregory, Busha et Tedesco, 2010, p. 67-69). L'intrigue est donc altérée, mais l'ordre et la structure des événements demeurent les mêmes. Enfin, à l'instar de De l'autre côté du miroir, Tales from Wonderland se termine avec un jeu d'échecs, qui rappelle la source d'inspiration pour les aventures d'Alice et certains des personnages au pays du miroir, tels le Roi et la Reine rouge. Dans le roman graphique, les auteurs tentent de forger ce lien entre les curieuses aventures qui se sont produites de l'autre côté d'un miroir et le jeu d'échecs en faisant référence au jeu que Elizabeth, la patiente enfermée à l'hôpital psychiatrique, a laissé derrière en s'enfuyant (Gregory, Busha et Tedesco, 2010, p. 129). Ainsi, même si nous faisons face à une tout autre 
histoire - un hypertexte ou une version transfictionnelle, voire contrefictionnelle, qui propose une autre lecture, beaucoup plus sombre, des curieux événements du pays du miroir-, les auteurs essaient quand même de respecter l'ordre général des aventures et de ponctuer le récit de repères ou de références qui renvoient directement au récit original et au monde ludique de Carroll. L'univers violent remplace l'univers farfelu et divertissant qui marquait l'œuvre originale dans le but de présenter une version « adulte » du conte.

\section{Once Upon a Time}

Enfin, la série télévisée américaine Once Upon a Time s'intéresse aussi à Alice au pays des merveilles et à ses différents personnages. Once Upon a Time est une série qui fonctionne comme un «forum transfictionnel » selon la terminologie de Saint-Gelais, c'est-à-dire une fiction dans laquelle il y a un rassemblement de héros et de vilains provenant de différentes histoires, particulièrement des contes de fées. Les personnages sont récupérés et mis en scène dans un même univers : la forêt enchantée. La série Once Upon a Time, comme les films de la série Shrek6, il nous semble, confirment le nouvel intérêt pour les «mondes des contes de fées »M qui deviennent de plus en plus populaires au cinéma et dans la littérature. En effet,

\footnotetext{
${ }^{6}$ Le premier film Shrek (2001) d'Andrew Adamson et de Vicki Jenson a d'ailleurs donné lieu à plusieurs suites, dont Shrek 2 (2004), Shrek the Third (2007) et Shrek Forever After. The Final Chapter (2010), de même qu'à une série «spin off », Puss in Boots (2011).
} 
quantité de films et de séries télévisées (La Maison des contes, Grimm), de romans graphiques (Fables, Grimm Fairy Tales) et d'ouvrages pour la jeunesse (Il était une fois au pays des contes, The Sisters Grimm, The Land of Stories) s'intéressent au phénomène des croisements. Plus particulièrement, dans Once Upon a Time, des épisodes se concentrent sur un personnage afin de montrer au téléspectateur comment ce dernier est devenu le personnage que nous connaissons si bien aujourd'hui. Pour ce faire, la série nous présente habituellement une autre version de son histoire célèbre, qui diffère de cette dernière et qui est remaniée de manière à tisser des liens avec les autres personnages féériques de la série. L'épisode, titré «Skin Deep » (Harowtiz et Kitsis, 2012), centré sur la Belle du conte de Madame Leprince de Beaumont, met notamment en scène Rumpelstiltskin comme la bête monstrueuse, mais aussi amoureuse.

Dans un des épisodes de la première saison, la série s'intéresse à l'un des personnages d'Alice au pays des merveilles: le Chapelier fou. Ici, la transfiction opère un recentrement puisqu'elle se concentre davantage sur un personnage qui était secondaire dans l'histoire initiale et elle laisse de côté Alice, la vraie héroïne du récit. L'épisode propose une "expansion parallèle », c'est-à-dire une autre version de l'histoire connue qui décrit ce que $x$ (le Chapelier fou) faisait pendant que l'histoire était centrée sur y (Alice) (Saint-Gelais, 2011, p. 93-94 et 183). Les créateurs de la série s'intéressent davantage au Chapelier, ce qui a pour effet de modifier la focalisation, d'altérer notre perception du récit de Carroll et d'élargir le monde imaginaire. 
En plus de donner plus d'importance au Chapelier fou, la transfiction complète l'histoire de Carroll en montrant au téléspectateur comment le Chapelier est arrivé au pays des merveilles et comment il est devenu fou. En regardant l'épisode, le téléspectateur apprend que le Chapelier, nommé Jefferson, était un père de famille qui habitait la forêt enchantée avec les autres personnages des contes de fées et qu'il jouait souvent à prendre le thé avec sa fille - un autre lien travaillé avec l'œuvre de Carroll. Jefferson était jadis un voyageur qui, avec son chapeau, pouvait ouvrir et fermer les portes entre les différents mondes imaginaires et faciliter le déplacement des habitants. Un jour, alors qu'il aidait la méchante reine de Blanche-Neige à voyager de la forêt enchantée au pays des merveilles, il fut trompé par cette dernière et il resta pris au pays des merveilles, incapable de revenir chez lui. C'est au pays des merveilles qu'il est devenu fabricant de chapeaux et, peu à peu, fou en essayant, tant bien que mal, de confectionner un nouveau chapeau magique qui pourrait le ramener chez lui auprès de sa fille. Par conséquent, puisque les créateurs de la série joignent une autre aventure à l'histoire du Chapelier, il y a un prequel dans cet épisode, ou ce que Gérard Genette appelle une «continuation analeptique» (1982, p.197). Selon Saint-Gelais, les prequels sont des récits qui «se dirigent vers une intrigue qui diégétiquement les suit, mais qui, en principe, les précède dans l'ordre de la lecture » (2011, p. 79-80). Assurément, c'est le cas ici puisque l'ajout des créateurs permet de préparer la scène pour Alice au pays des merveilles, que plusieurs lecteurs ou téléspectateurs connaissent déjà. Dans l'épisode de Once Upon a Time portant sur Alice au pays des merveilles, il y alors un recentrement du récit initial, puisque nous nous intéressons davantage à l'histoire du Chapelier fou, et un prequel, c'est-à- 
dire un récit en amont de l'intrigue principale, puisque l'épisode est surtout axé sur le parcours du Chapelier avant qu'il vienne au pays des merveilles. L'épisode explore ainsi l'histoire du Chapelier fou et d'Alice au pays des merveilles sous un autre angle et élargit le monde imaginaire conçu par Carroll.

En somme, il est clair que chacune des œuvres étudiées peint une différente Alice et met de l'avant une autre facette de l'héroïne et de son histoire afin de recréer le personnage, le pays des merveilles ou le pays du miroir pour un auditoire particulier et de consolider leur place dans l'imaginaire. Malgré les nombreuses reprises, les aventures de la fillette demeurent intéressantes puisqu'elles encouragent le déploiement de l'imaginaire et la création de nouveaux mondes. Les univers ludiques de Carroll, qui se dessinent comme des carrefours de la tradition anglaise puisqu'ils recyclent des personnages qui circulent déjà dans la culture populaire, stimulent aussi l'imagination en promouvant eux-mêmes la reprise et le renouvellement de héros ainsi que le croisement entre diverses histoires et différents mondes fictionnels. Comme elle provient de romans qui ont joui d'un grand et d'un long succès et comme le voyage lui est associé dans ses romans initiaux, Alice, de même que les autres héros du pays des merveilles et du pays du miroir, devient un personnage extrêmement malléable qui parvient facilement à se déplacer vers de nouveaux genres littéraires et à s'adapter à de nouvelles diégèses. C'est ainsi qu'elle continue à se façonner une place permanente dans l'imaginaire collectif : en se transformant et en se modernisant, mais en demeurant toujours la petite fille libre, curieuse et rêveuse. 


\section{Bibliographie}

BEnNETT, Tony et Janet WoollacotT. (1987), Bond and Beyond: The Political Career of a Popular Hero, New York, Methuen.

Belmont, Nicole. (1999), Poétique du conte, Paris, Gallimard.

BouSlOUGH, Gail. (2007), Appropriating Wonderland: Nostagia and Modernity in the Children's Fantasy of Lewis Carroll's Alice books and L. Frank Baum's The Wonderful Wizard of Oz, Claremont, Claremont Graduate University.

Carroll, Lewis. (1990), Alice au pays des merveilles. De l'autre côté du miroir [édition présentée et annotée par Jean Gattégno], Jacques Papy (trad.), Paris, Gallimard, coll. « Folio classique ».

CARroll, Lewis. (1998), Alice's Adventures in Wonderland and Through the Looking-Glass, Londres, Penguin, coll. «Classics ».

DAUNAIS, Isabelle. (2007), «Condition du personnage transfictionnel », dans Richard Saint-Gelais et René Audet (dir.), La Fiction, suites et variations, Québec, Nota bene, p. 349-368.

ESCARPIT, Denise. (2008), La «littérature de jeunesse. Itinéraire d'hier à aujourd'hui, Paris, Magnard.

GenetTe, Gérard. (1982), Palimpsestes. La littérature au second degré, Paris, Seuil, coll. « Poétique ».

GudulE. (1995), La Bibliothécaire, Paris, Hachette, coll. « Le livre de poche jeunesse ».

HALBWACHS, Maurice. (1950), «La mémoire collective et le temps ", dans La Mémoire collective, Paris, Presses universitaires de France, p. 80-129.

HaRowTIZ, Adam et Edward Kitsis. (2012), «Skin Deep », Once Upon a Time, États-Unis ABC, no 12. 
-. (2012), «Hat Trick», Once Upon a Time, États-Unis ABC, no 17 .

OPIE, Iona et Peter. (1984), The Oxford Nursery Rhyme Book, Oxford, Oxford University Press.

PoPOVIC, Pierre. (2008), «Introduction », dans Imaginaire social et folie littéraire. Le second Empire de Paulin Gagne, Montréal, Les Presses de l'Université de Montréal, coll. "Socius", p. 11-33.

PRINCE, Nathalie (2010), La Littérature de jeunesse. Pour une théorie littéraire, Paris, A Colin.

Raven, Gregory, Joe Brusha et Ralph Tedesco. (2010), Tales from Wonderland. Volume II, Fort Washington, Zenescope.

SAINT-GELAIS, Richard. (2001), Fictions transfuges. La transfictionnalité et ses enjeux, Paris, Seuil, coll. «Poétique ».

\section{Résumé}

Connue pour ses aventures au pays des merveilles, Alice est un personnage du XIX ${ }^{\text {e }}$ siècle qui, encore aujourd'hui, continue de jouir d'un succès littéraire et culturel. Dans cette étude, nous analyserons l'histoire et le personnage d'Alice en vue d'extraire la quintessence $\mathrm{du}$ personnage transfictionnel. Nous dégagerons d'abord les conditions du personnage transfictionnel, c'est-à-dire les caractéristiques de l'héroïne et de ses livres qui facilitent les reprises. Puis, dans une deuxième partie, nous regarderons diverses transfictions contemporaines qui s'intéressent à la fillette afin de comprendre comment son histoire a été reprise par différents auteurs et transformée pour des publics variés. 


\begin{abstract}
Known for her adventures in Wonderland, Alice is a character of the XIX ${ }^{\text {th }}$ century that, still today, continues to have a literary and cultural success. In this study, we will analyse the Alice character as well as her stories in order to find the essence of the transfictional character. We will firstly extract the conditions of the transfictional character, namely the heroin's and the books' characteristics that facilitate reprisals. Then, in a second part, we will study contemporary transfictions that recapture Alice's story, in order to understand how her story has been rewritten by various authors and transformed for various publics.
\end{abstract}

